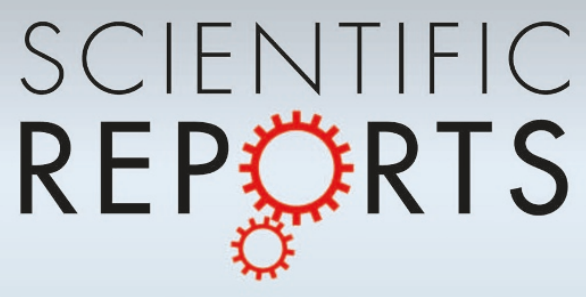

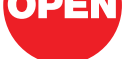

SUBJECT AREAS:

IMAGING

QUANTUM CHEMISTRY

APPLIED PHYSICS

INFORMATION THEORY AND COMPUTATION

Received

8 June 2012

Accepted

7 August 2012

Published

20 August 2012

Correspondence and requests for materials should be addressed to

D.S. (dominique. sugny@u-bourgogne. fr) or S.J.G. (glaser@ tum.de)

\section{Exploring the Physical Limits of Saturation Contrast in Magnetic Resonance Imaging}

\author{
M. Lapert' ${ }^{2}$, Y. Zhang ', M. A. Janich' , S. J. Glaser' \& D. Sugny ${ }^{2}$
}

'Department of Chemistry, Technische Universität München, Lichtenbergstrasse 4, D-85747 Garching, Germany, ${ }^{2}$ Laboratoire Interdisciplinaire Carnot de Bourgogne (ICB), UMR 5209 CNRS-Université de Bourgogne, 9 Av. A. Savary, BP 47870 , F-21078 DIJON Cedex, FRANCE.

Magnetic Resonance Imaging has become nowadays an indispensable tool with applications ranging from medicine to material science. However, so far the physical limits of the maximum achievable experimental contrast were unknown. We introduce an approach based on principles of optimal control theory to explore these physical limits, providing a benchmark for numerically optimized robust pulse sequences which can take into account experimental imperfections. This approach is demonstrated experimentally using a model system of two spatially separated liquids corresponding to blood in its oxygenated and deoxygenated forms.

ince its discovery in the forties, Nuclear Magnetic Resonance (NMR) has become a powerful tool ${ }^{1,2}$ to study the state of matter in a variety of domains extending from biology and chemistry ${ }^{3}$ to solid-state physics and quantum computing ${ }^{4,5}$. The power of NMR techniques is maybe best illustrated by medical imaging ${ }^{6}$, where it is possible e.g. to produce a three-dimensional picture of the human brain. NMR spectroscopy and Magnetic Resonance Imaging (MRI) involve the manipulation of nuclear spins via their interaction with magnetic fields. All experiments in liquid phase can be described in a first approach as follows. A sample is held in a strong and uniform longitudinal magnetic field denoted $B_{0}$. The magnetization of the sample is then manipulated by a particular sequence of transverse radio-frequency magnetic pulses $B_{1}$ in order to prepare the system in a particular state. The analysis of the radio-frequency signal that is subsequently emitted by the nuclear spins leads to information about the structure of the molecule and its spatial position. One deduces from this simple description that the crucial point of this process is the initial preparation of the sample, i.e. to design a corresponding pulse sequence to reach this particular state with maximum efficiency. The maximum achievable efficiency can be determined for the transfer between well defined initial and target states ${ }^{7}$ if relaxation effects can be neglected. In imaging applications, where relaxation forms the basis for contrast, a very large number of different strategies have been proposed and implemented so far with the rapid improvement of NMR and MRI technology ${ }^{2,6}$. However, there was no general approach to provide the maximum possible performance and the majority of these pulse sequences have been built on the basis of intuitive and qualitative reasonings or on inversion methods such as the Shinnar-Le Roux algorithm ${ }^{8}$. Note that this latter can be applied only in the case where there is no relaxation effect and radio-frequency inhomogeneity.

A completely different point of view emerges if this problem is approached from an optimal control perspective. Optimal control theory was created in its modern version at the end of the 1950s with the Pontryagin Maximum Principle (PMP) $)^{9-11}$. Developed originally for problems in space mechanics, optimal control has become a key tool in a large spectrum of applications including engineering, biology and economics. Solving an optimal control problem leads to the determination of a particular trajectory, that is a solution of an associated Hamiltonian system constructed from the PMP and satisfying given boundary conditions. This approach has found remarkable applications in quantum computing and NMR spectroscopy, but its application to MRI has been limited to the numerical design of slice-selective $90^{\circ}$ and $180^{\circ}$ pulses ${ }^{12}$.

Despite the efficiency of MRI techniques currently used in clinics, some aspects still pose fundamental problems of both theoretical and practical interest. The enhancement of contrast remains one of the crucial questions for improving image quality and the corresponding medical diagnosis. The use of particular pulse sequences to generate image contrast based on relaxation rates is not new in MRI, since this question was raised at the beginning of the development of MRI in the 1970s. Different strategies have been proposed, such as the Inversion Recovery sequence ${ }^{13,14}$ for $T_{1}$ contrast and pulses for ultra short echo time experiments for $T_{2}$ contrast $^{15}$ (See Eq. (1) for the definition of $T_{1}$ and $T_{2}$ parameters). Here, we go beyond such intuitive methods by using the powerful machinery of optimal control, which provides in this case not just an improved performance but an 
estimate of the global optimum, i.e. the best possible contrast within the experimental constraints (see the supplementary material for mathematical details). This optimized contrast is demonstrated in a laboratory benchmark experiment.

In its simplest form, the contrast problem can be stated by assuming that the signal is composed of two different contributions. We consider as a benchmark example the case of (a) oxygenated vs. (b) deoxygenated blood, where the spins that are probed are the ones of the hydrogen atoms of water $\left(\mathrm{H}_{2} \mathrm{O}\right)$. This is e.g. an important issue in functional studies of the human brain. These spins have different relaxation rates due to the interaction with other molecules such as hemoglobin in its oxygenated or non-oxygenated form, leading thus to two different signatures of the relaxation dynamics of the magnetization, which is governed by the Bloch equations:

$$
\left\{\begin{array}{l}
\frac{d M_{x}^{i}}{d t}=-\omega M_{y}^{i}+\omega_{y} M_{z}^{i}-M_{x}^{i} / T_{2}^{i} \\
\frac{d M_{y}^{i}}{d t}=\omega M_{x}^{i}-\omega_{x} M_{z}^{i}-M_{y}^{i} / T_{2}^{i} \\
\frac{d M_{z}^{i}}{d t}=-\omega_{y} M_{x}^{i}+\omega_{x} M_{y}^{i}-\left(M_{z}^{i}-M_{0}\right) / T_{1}^{i},
\end{array}\right.
$$

where $\vec{M}^{i}=\left(M_{x}^{i}, M_{y}^{i}, M_{z}^{i}\right)$ is the magnetization vector considered with $i=(a, b), M_{0}$ is the equilibrium magnetization, $T_{1}^{i}$ and $T_{2}^{i}$ the longitudinal and transverse relaxation rates, $\omega$ the resonance offset and $\omega_{x}$ and $\omega_{y}$ the components in a rotating frame of the transverse magnetic field along the $x$ - and $y$ - directions. While different definitions of contrast exist in the literature ${ }^{6}$, here we consider a particular case that we call the saturation contrast, where the objective of the control problem is to find the pulse sequence which completely suppresses the contribution of one of the two magnetization vectors while maximizing the modulus of the other. If such a pulse module can be found, it can be used in combination with a large number of possible host sequences for imaging and spectroscopy (see Chapter 14 of Ref. [6] for details).

\section{Results}

In this section, we first analyze the ideal situation of a homogeneous ensemble of spin $1 / 2$ particles irradiated on resonance, which is described by Eq. (1) with $\omega=0$. We introduce the normalized vectors $\vec{V}^{i}=\vec{M}^{i} / M_{0}$ with coordinates $\left(X^{i}, Y^{i}, Z^{i}\right)$ to eliminate the equilibrium magnetization $M_{0}$. Based on numerical computations (see also ${ }^{16-18}$ for analytical details), we restrict, without loss of generality, the dynamics to a meridian plane by assuming $\omega_{y}=0$. Using advanced techniques of geometric optimal control theory ${ }^{10,19}$, it is possible to find the desired control field $\omega_{x}(t)$ which provides the optimum contrast by a direct solution of the PMP. The details of the theoretical approach are given in Section 1 of the supplementary material.

As a test case, we chose the typical relaxation parameters for (a) oxygenated and (b) deoxygenated blood with identical $T_{1}$ values ( $\left.T_{1}^{a, b}=1.35 \mathrm{~s}\right)$ but different $T_{2}$ values $\left(T_{2}^{a}=200 \mathrm{~ms}, T_{2}^{b}=50 \mathrm{~ms}\right)$. Note that in this situation, the conventional Inversion Recovery experiment, which relies on $T_{1}$ differences cannot provide useful contrast. However, applying the optimal control approach for these parameters the maximum of the modulus of $\vec{V}^{a}$ (under the condition that $\left|\vec{V}^{b}\right|=0$ ) is found to be $\left|\vec{V}^{a}\right|=0.4663$, representing the maximum achievable saturation contrast in this system. Note that a similar computation can be done to saturate the spin (a) and maximize $\left|\vec{V}^{b}\right|$, with a final result of $\left|\vec{V}^{b}\right|=0.4731$. We underline that this saturation contrast is optimal in the sense that it is the physical upper limit that can be reached within the experimental constraints given here by the values of the relaxation rates. The shape of the optimal pulse is shown in Fig. 1a. For our demonstration experiments, we did not use actual blood samples but prepared two different solutions with similar physical characteristics (see the Methods section). As the experimental $T_{2}^{*}$ values ${ }^{2}$ of the test sample were only about $2 / 3$ of the $T_{2}$ values assumed for the optimized pulse, the pulse duration and amplitude were scaled by $2 / 3$ and $3 / 2$, respectively, resulting in a scaled pulse duration of $0.214 \mathrm{~s}$ and a maximum pulse amplitude of the order of $10 \mathrm{~Hz}$ (see Fig. 1a). Within the experimental accuracy, we have checked that, in this case, this scaling procedure provides the same contrast as the optimal solution. The optimal control field was implemented experimentally as a shaped pulse on a standard Bruker Avance III $600 \mathrm{MHz}$ spectrometer and the experimental trajectories were measured using two different samples in different test tubes in order to approach ideal experimental conditions with negligible magnetic field inhomogeneities. Under these conditions, the dynamics is described with high accuracy by the Bloch equations (1). The simulated and experimental trajectories of the two magnetization vectors $\vec{V}^{a}(t)$ and $\vec{V}^{b}(t)$ are shown in Fig. $1 \mathrm{~b}$ and a good match is found between theory and experiment.

So far, we assumed that there is no experimental imperfection due to magnetic field inhomogeneities, which are however not negligible in realistic imaging experiments and have therefore to be taken into account. Nevertheless, it is important to point out that the analytical PMP-based optimal solution in the absence of experimental imperfections gives an estimate of a previously unavailable physical upper limit of the maximum achievable saturation contrast, the inhomogeneities having a detrimental effect on the final result. This bound, which can be determined for any set of relaxation parameters, is thus relevant for any imaging contrast problem. Hence, it gives a fundamental benchmark to assess the performance of standard methods and numerically optimized pulse sequences in this domain.
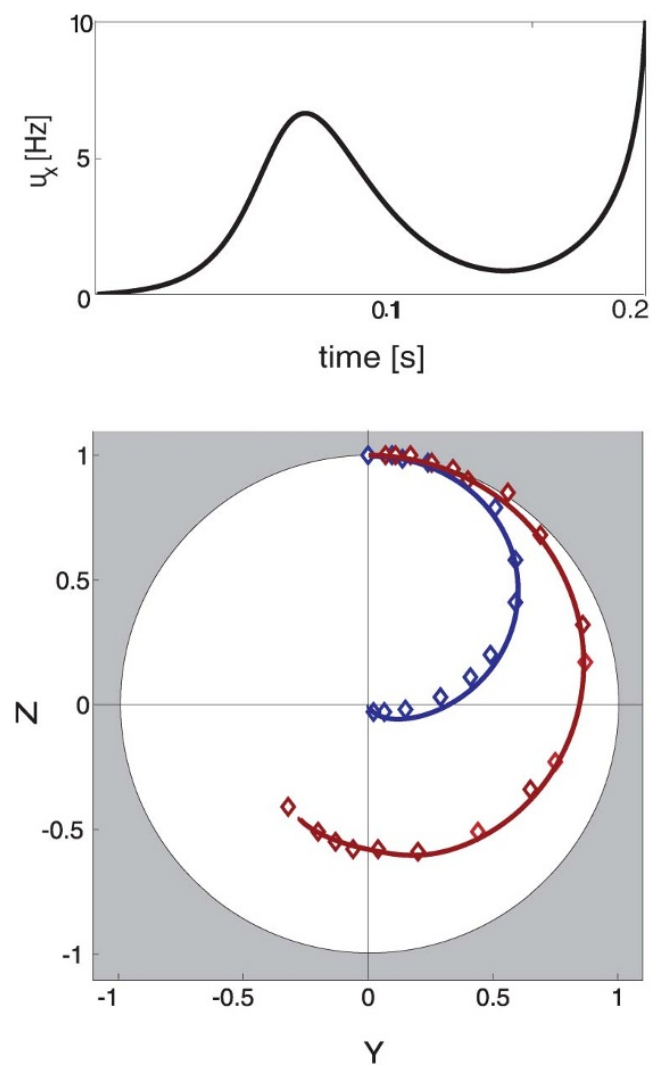

Figure $1 \mid$ Optimal pulse sequence and trajectories for negligible $B_{0}$ and $B_{1}$ inhomogeneities. (a), The control amplitude $u_{x}(t)=\omega_{x}(t) /(2 \pi)$ is shown for the optimal pulse sequence to maximize $\left|\vec{V}^{a}\right|$ under the condition that $\left|\vec{V}^{b}\right|=0$. (b), Corresponding simulated (solid curves) and experimental (open diamonds) trajectories of $\vec{V}^{a}(t)$ (red) and $\vec{V}^{b}(t)$ (blue) in the $(Y, Z)$ plane for a homogeneous ensemble of spin particles. 


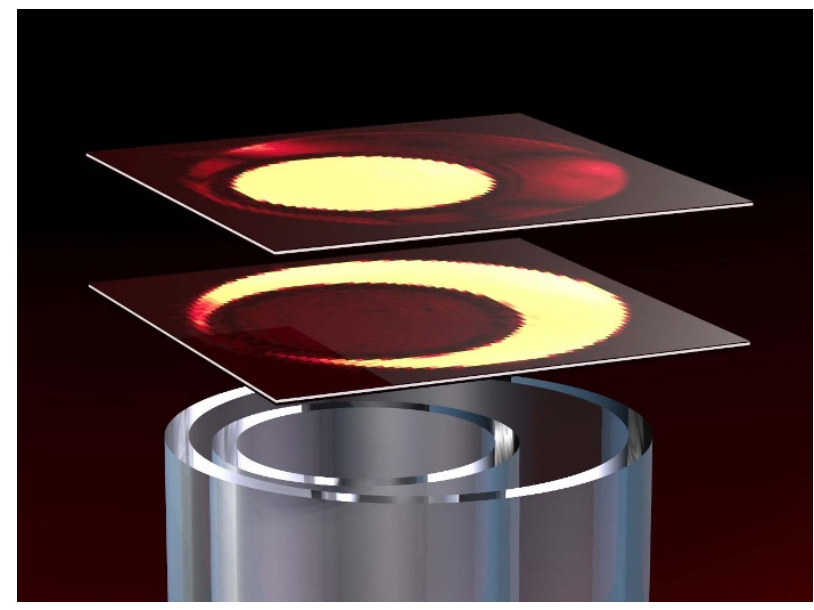

Figure $2 \mid$ Geometry of the test sample used for the imaging experiments. In the micro imaging experiments, the sample consists of two test tubes with outer diameters of $5 \mathrm{~mm}$ and $8 \mathrm{~mm}$. The outer and inner volumes were respectively filled with the two solutions corresponding to (a) oxygenated and (b) deoxygenated blood. The two slices represent the experimental results after the saturation of the samples (a) (top) and (b) (bottom). (see also the results of Fig. 4)

To perform a realistic imaging experiment, we designed a test sample consisting of a small test tube in a larger tube with an outer diameter of $8 \mathrm{~mm}$, forming two compartments filled with solutions (a) and (b) corresponding to the relaxation rates of deoxygenated and oxygenated blood, see Fig. 2 for a schematic representation. The experiments were performed using the same spectrometer as described above, equipped with a micro-imaging unit. The details are given in Section 2 of the supplemental material. Figure 3 shows the experimentally measured spatial $B_{0}$ and $B_{1}$ distributions in the central slice of the sample. The variation of $B_{0}$ corresponds to resonance frequency shifts $\omega$ between 0 and $-30 \mathrm{~Hz}$, while the experimental scaling of the $B_{1}$ field (which is proportional to the control amplitude) is $\pm 20 \%$.

In general, the frequency offsets created by the $B_{0}$ inhomogeneities are negligible if they are dominated by the amplitude of the control field (in units of $\mathrm{Hz}$ ). However, the control amplitude of the analytically optimal pulse sequence shown in Fig. 1a is less than $10 \mathrm{~Hz}$, which is smaller than the resonance offset variation due to $B_{0}$ inhomogeneities (see Fig. 3a) and therefore, the optimal sequence derived analytically for an ideal case is not expected to work in the experimental micro imaging setting. In addition, the experimental variation of $B_{1}$ scaling was not considered in the analytical solution and is also expected to have detrimental effects on the pulse performance.

In order to take into account the experimentally measured $B_{0}$ and $B_{1}$ distributions, numerically optimized pulse sequences were computed with the GRAPE algorithm ${ }^{20}$, which is a standard iterative algorithm to solve the optimization equations. The pulses are designed to work for an ensemble of spins approximately within the range of the $B_{0}$ and $B_{1}$ inhomogeneities experimentally measured. In the numerical optimizations, we fixed the pulse duration to the duration of the corresponding analytical pulse. Compared to the fundamental contrast benchmark $\left(\left|V^{a}\right|=0.47\right.$ and $\left.\left|V^{b}\right|=0\right)$ provided by the PMP-based analytical solution, the minimum value (worst case) of $\left|V^{a}\right|$ for the considered range of $B_{0}$ and $B_{1}$ inhomogeneities is 0.37 (that is $79 \%$ of the physically maximum saturation contrast achievable) while the maximum value (worst case) of the incompletely suppressed $\left|V^{b}\right|$ is 0.054 . Conversely, when the goal is to saturate spin (a) and to maximize the magnetization vector of spin (b), the optimal pulse sequence yields $\left|\vec{V}^{a}\right|=0.047$ and $\left|\vec{V}^{b}\right|=0.33$. Figure 4 shows resulting experimental images which are in good
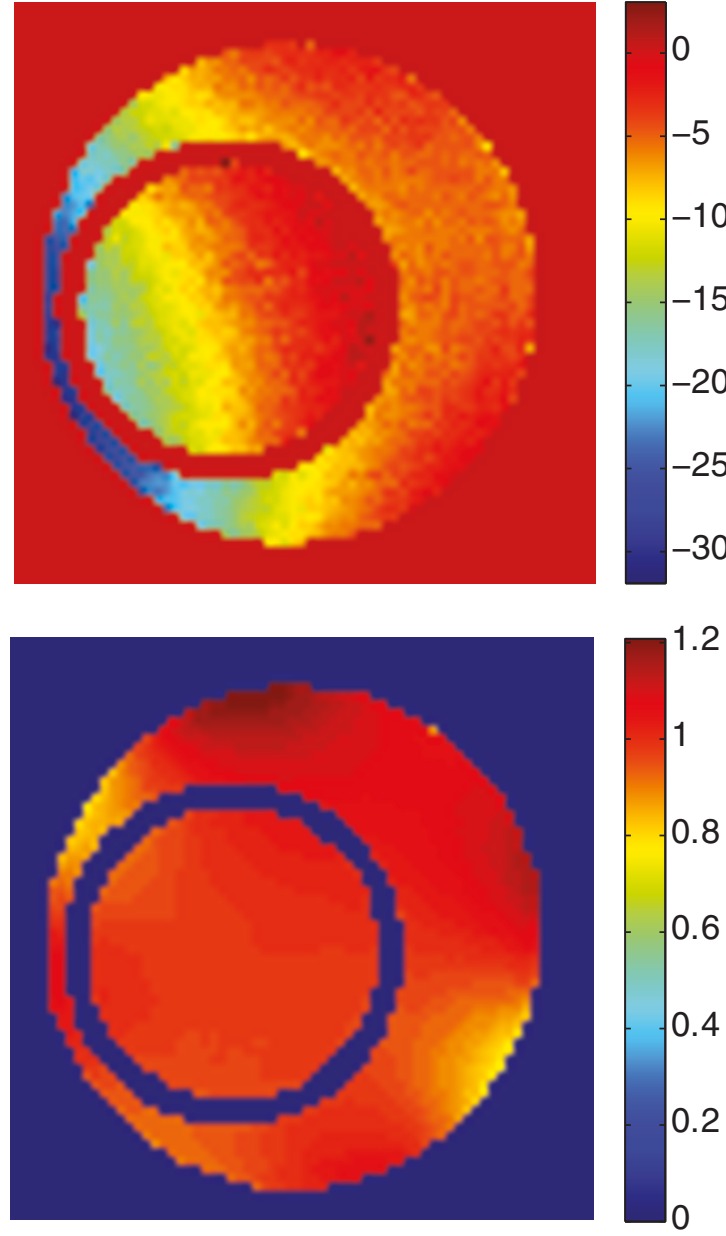

Figure 3 Experimental spatial $B_{0}$ and $B_{1}$ distributions. Spatial distributions of the $B_{0}(\mathrm{a})$ and $B_{1}(\mathrm{~b})$ amplitudes in the central slice of the sample. The $B_{0}$ variation is represented by the corresponding ${ }^{1} \mathrm{H}$ frequency offsets in units of $\mathrm{Hz}$ as a function of the spatial position, while the $B_{1}$ variation is described by a dimensionless scaling factor.

agreement with simulated data (see the supplementary material for the details of the computation).

\section{Discussion}

We demonstrated to which extend saturation contrast based on different relaxation times $T_{1}$ and $T_{2}$ can be maximized in magnetic resonance imaging within given experimental constraints. Starting from the analytic optimal solution of the homogeneous case, we have then designed a particular pulse sequence using numerical tools of optimal control theory to approach in a realistic experiment this physical limit. We emphasize that one of the main advantages of this contrast enhancement is its general character since the optimal control fields can be computed with standard routines published in the literature and implemented on a standard NMR spectrometer without requiring specific materials and process techniques.

The efficiency of this approach was shown in a laboratory experiment using a model system for the relaxation parameters of deoxygenated and oxygenated blood. The presented method fully exploits the differences of both $T_{1}$ and $T_{2}$ to create the maximum possible saturation contrast as opposed to conventional approaches based on $T_{1}$ or $T_{2}$ differences. The combined analytical and numerical optimal control approach is not limited to the definition of saturation contrast (motivated by typical magnitude mode imaging experiments) used here for demonstration, but can also be applied to more general definitions of relaxation-based contrast, e.g. for phase-sensitive 

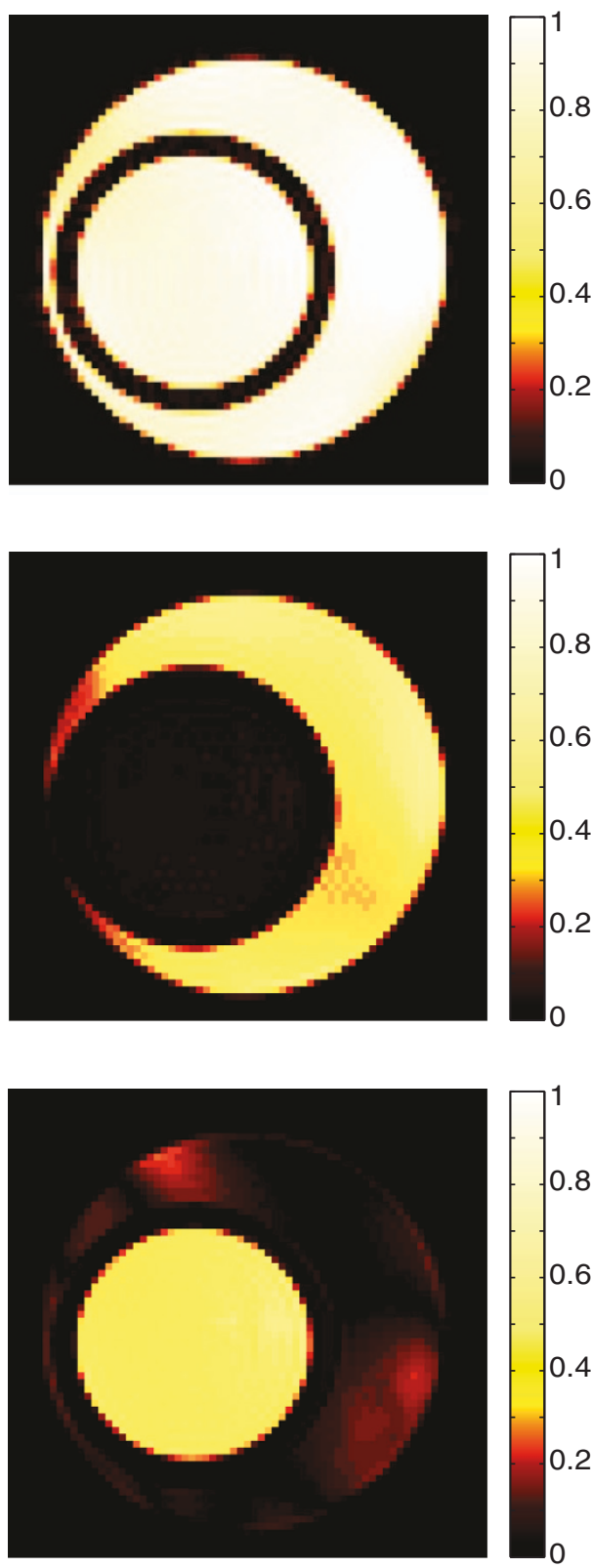

Figure 4 Experimental implementation of the robust optimal pulse. Optimization of the contrast when the deoxygenated (b) or the oxygenated (c) blood is saturated. Figure (a) displays the reference image after the application of a 90 degree pulse.

images and for a wide variety of possible host imaging sequences that can be applied after the contrast pulse module ${ }^{6}$. Furthermore, the flexibility of the optimal control approach makes it possible to include experimental constraints such as bounds on the control amplitude or pulse energy or non-linear effects such as radiation damping ${ }^{21}$.

We expect that the presented principles will find practical applications in MRI and in particular in medical imaging, where increased contrast and sensitivity could not only help in the diagnosis but could also reduce the required concentration of commonly used contrast agents, which could be beneficial to the patient.

\section{Methods}

Experimental sample. The relaxation properties of oxygenated blood were approached by solution (a) consisting of $90 \% \mathrm{D}_{2} \mathrm{O}, 10 \%$ glycerol and doped with $\mathrm{CuSO}_{4}$ with relaxation times $T_{1}^{a}=1.8 \mathrm{~s}, T_{2}^{a}=260 \mathrm{~ms}$ (as determined from
CPMG-experiment) and $T_{2}^{*, a}=100 \mathrm{~ms}$ (as determined from the experimental line width). Deoxygenated blood was modeled by solution (b) consisting of $70 \% \mathrm{D}_{2} \mathrm{O}, 30 \%$ glycerol and doped with $\mathrm{CuSO}_{4}$ with $T_{1}^{b}=1.4 \mathrm{~s}, T_{2}^{b}=60 \mathrm{~ms}$ and $T_{2}^{*, b}=30 \mathrm{~ms}$.

Measurement method of the $B_{0}$ and $B_{1}$ field maps. Mapping of local $B_{0}$ offsets was accomplished by evaluating the signal phase evolution between two echoes acquired in a dual-echo gradient pulse sequence ${ }^{6}$. The echo times of the $3 \mathrm{D}$ image acquisition were $T E_{1}=1.5 \mathrm{~ms}$ and $T E_{2}=11.5 \mathrm{~ms}$. Figure $3 \mathrm{a}$ of the main text shows the $B_{0}$ field map in the central axial slice. The amplitude of the $B_{1}$ excitation field was measured by using the cosine-like dependence of the remaining signal after a saturation pulse and fitting to a curve measured with multiple saturation flip angles ${ }^{22}$. The applied saturation flip angles were $10^{\circ}, 20^{\circ}, \ldots, 300^{\circ}$. Robust $B_{1}$ mapping was achieved by fitting a signal model to the acquired slice-selective gradient echo signal in a linear least-squares sense.

Application of the optimized pulse sequence. For the imaging experiment, the $\mathrm{H}_{2} \mathrm{O}$ content was increased in the sample in order to have a sufficient signal-to-noise ratio. The outer and inner volumes of the sample were filled with the following solutions: "oxy sample II" $\left(80 \% \mathrm{D}_{2} \mathrm{O}, 10 \% \mathrm{H}_{2} \mathrm{O}, 10 \%\right.$ glycerol doped with $\mathrm{CuSO}_{4}$ with relaxation times of $T_{1}=2.6 \mathrm{~s}$ and $\left.T_{2}^{*}=100 \mathrm{~ms}\right)$ and "deoxy sample II" $\left(60 \% \mathrm{D}_{2} \mathrm{O}, 10 \% \mathrm{H}_{2} \mathrm{O}, 30 \%\right.$ glycerol doped with $\mathrm{CuSO}_{4}$ with relaxation times of $T_{1}=1.4 \mathrm{~s}$ and $T_{2}^{*}=30 \mathrm{~ms}$ ). The optimal pulses were implemented into a gradient echo pulse sequence ${ }^{23}$ without slice selection. The experiments were performed with a $600 \mathrm{MHz}$ Bruker Avance III spectrometer equipped with a micro-imaging unit. The field of view (FOV) was $15 \mathrm{~mm} \times 15 \mathrm{~mm}$, and in the third dimension it was limited to approximately $10 \mathrm{~mm}$ by coil sensitivity. The repetition time (TR) is $7 \mathrm{~s}$ and the echo time (TE) is $2 \mathrm{~ms}$ with the matrix size $128 \times 128$. Figures S1 and S2 of the supplementary material show simulated (d) and experimental (e) images using the optimized pulse to saturate the "deoxy sample II" in the inner cylinder (Fig. S1) and for the optimized pulse to saturate the "oxy sample II" in the outer cylinder (Fig. S2).

1. Ernst, R. R. Principles of Nuclear Magnetic Resonance in one and two dimensions (International Series of Monographs on Chemistry, Oxford University Press, Oxford, 1990).

2. Levitt, M. H. Spin dynamics: basics of nuclear magnetic resonance (John Wiley and sons, New York-London-Sydney, 2008).

3. Bhattacharya, A. Chemistry: Breaking the billion-hertz barrier. Nature 463, 605 (2010).

4. Chuang, I., Vandersypen, L., Zhou, X., Leung, D. \& Lloyd, S. Experimental realization of a quantum algorithm. Nature 393, 344 (1998).

5. Vandersypen, L. M. K. \& Chuang, I. L. NMR techniques for quantum control and computation. Rev. Mod. Phys. 76, 1037 (2005).

6. Bernstein, M. A., King, K. F. \& Zhou, X. J. Handbook of MRI Pulse Sequences (Elsevier, Burlington-San Diego-London, 2004).

7. Glaser, S. J. et al. C. Unitary Control in Quantum Ensembles, Maximizing Signal Intensity in Coherent Spectroscopy. Science 280, 421 (1998).

8. Pauly, J., Le Roux, P., Nishimura, D. \& Macovski, A. Parameter relations for the Shinnar-Le Roux selective excitation pulse design algorithm. IEEE Trans. Med. Imag. 10, 53 (1991).

9. Pontryagin, L. et al. Mathematical theory of optimal processes (Mir, Moscou, 1974).

10. Bonnard, B. \& Chyba, M. Singular trajectories and their role in control theory (Springer SMAI, Vol. 40, 2003).

11. Jurdjevic, V. Geometric control theory (Cambridge University Press, Cambridge, 1996).

12. Nielsen, N. C., Kehlet, C., Glaser, S. J. \& Khaneja, N. Optimal control methods in NMR spectroscopy (Encyclopedia of Nuclear Magnetic Resonance, Wiley, 2010).

13. Bydder, G. M. \& Yound, I. R. MR imaging: clinical use of the inversion recovery sequence. Journal of computed assisted tomography 9, 659 (1985).

14. Bydder, G. M., Hajnal, J. V. \& Young, I. R. MRI: Use of the inversion recovery pulse sequence. Clinical radiology 53, 159 (1998).

15. Carl, M., Bydder, M., Du, J., Takahashi, A. \& Han, E. Optimization of RF excitation to maximize signal and $T_{2}$ contrast of tissues with rapid transverse relaxation. Magnetic resonance in medicine 64, 481 (2010).

16. Bonnard, B. \& Sugny, D. Time-minimal control of dissipative two-level quantum systems: The integrable case. SIAM J. on Control and Opt.48, 1289 (2009).

17. Bonnard, B. Chyba, M. \& Sugny, D. Time-minimal control of dissipative two-level quantum systems: The generic case. IEEE Trans. Autom. Control 54, 2598 (2009).

18. Bonnard, B. et al. Geometric optimal control of the contrast imaging problem in Nuclear Magnetic Resonance. IEEE Trans. Autom. Control 57, 1957 (2012).

19. Lapert, M., Zhang, Y., Braun, M., Glaser, S. J. \& Sugny, D. Singular extremals for the time-optimal control of dissipative spin 1/2 particles. Phys. Rev. Lett. 104, 083001 (2010).

20. Skinner, T. E., Reiss, T. O., Luy, B., Khaneja, N. \& Glaser, S. J. Application of Optimal Control Theory to the Design of Broadband Excitation Pulses for High Resolution NMR. RJ. Magn. eson. 163, 8 (2003).

21. Zhang, Y., Lapert, M., Braun, M., Sugny, D. \& Glaser, S. J. Timeoptimal control of spin $1 / 2$ particles in presence of relaxation and radiation damping effects. J. Chem. Phys. 134, 054103 (2011).

22. Brunner, D. O. \& Pruessmann, K. P. $\mathrm{B}_{1}{ }^{+}$interferometry for the calibration of RF transmitter arrays. Magn. Reson. Med. 61, 1480 (2009). 
23. Frahm, J., Haase, A. \& Matthaei, D. Rapid NMR imaging of dynamic processes using the FLASH technique. Magn. Reson. Med. 3, 321 (1986).

\section{Acknowledgment}

We are grateful to B. Bonnard and H. R. Jauslin for discussions and Franz Schilling for help in the imaging experiments. S.J.G. acknowledges support from the DFG (GI 203/6-1), SFB 631. S. J. G. thanks the Fonds der Chemischen Industrie. Experiments were performed at the Bavarian NMR center at TU München.

\section{Author contributions}

All authors contributed to the design and interpretation of the presented work. Numerical computations have been done by M. L. and Y. Z., the construction of the experiment and to the acquisition of the data was performed by Y. Z., M. A. J. and S. J. G.

\section{Additional information}

Supplementary information accompanies this paper at http://www.nature.com/ scientificreports

Competing financial interests: The authors declare no competing financial interests. License: This work is licensed under a Creative Commons

Attribution-NonCommercial-ShareAlike 3.0 Unported License. To view a copy of this license, visit http://creativecommons.org/licenses/by-nc-sa/3.0/

How to cite this article: Lapert, M., Zhang, Y., Janich, M.A., Glaser, S.J. \& Sugny, D.

Exploring the Physical Limits of Saturation Contrast in Magnetic Resonance Imaging. Sci. Rep. 2, 589; DOI:10.1038/srep00589 (2012). 